\title{
Una aproximación a la entrevista en investigaciones sobre música
}

\author{
Nancy Marcela Sánchez \\ [Facultad de Artes y Ciencias Musicales - \\ Universidad Católica Argentina]
}

RESUMEN En este artículo problematizo la
entrevista como técnica de recolección de
información, como encuentro etnográfico y
como proceso de producción de textualidad.
Propongo un marco teórico adecuado para exa-
minar los roles que los participantes asumen,
las relaciones de poder social y las negocia-
ciones que se llevan a cabo.
Desarrollo conceptos tales como objetivos inte-
raccionales, situación social, marco, entextua-
lización de los discursos y contextualización de
las prácticas de performance, que proporcio-
nan una fundamentación apropiada para el
análisis de los datos recabados. Comparo dis-
tintos tipos de entrevista, diferencio sus pro-
pósitos y señalo la relación que cada formato
tiene con el diseño de las investigaciones (cua-
litativa, cuantitativa, mixta). Los ejemplos ayu-
dan a pensar la planificación de las acciones
que se llevarán adelante en las entrevistas e
informan sobre los problemas que pueden sur-
gir en el proceso. Hago énfasis en la revisión de
las nociones de contexto y contextualización.

Palabras clave: entrevista .

contextualización · relaciones de poder
Summary In this article I problematize the interview as an information gathering (data collection) technique, as an ethnographic encounter and as a textuality production process. I propose an adequate theoretical framework to examine the roles that the participants assume, the social power relations and the negotiations that take place.

I develop concepts such as interactional objectives, social situation, and entextualization of discourses and contextualization of performance practices, which provide an adequate foundation for the analysis of the data collected. I compare different types of interview, differentiate their purposes and point out the relationship that each format has with the research design (qualitative, quantitative, mixed methods). The examples help to think about the planning of the actions that will be carried out in the interviews and inform about the problems that may arise in the process. I emphasize the revision of the notions of context and contextualization.

Keywords: interview · contextualization · social power relationships 


\section{INTRODUCCIón}

El presente artículo trata sobre las entrevistas en el marco de proyectos de investigación llevados a cabo por profesionales del campo de la música o estudiantes avanzados que se inician en esta práctica.

La entrevista cuenta con una extensa historia de conceptualización en los estudios de folklore (narrativa oral), sociolingüística, etnografía del habla y teoría literaria (Ben Amos, I995; Briggs, I986; Bauman y Briggs, 1996). La convergencia de estas disciplinas en el folklore norteamericano dio por resultado la elaboración de una plataforma teóricometodológica que contribuyó a repensar lo que acontece en la entrevista. Desde este enfoque, realizo una aproximación a la entrevista entendida como el encuentro etnográfico entre quienes representan a las instituciones académicas o científicas (los investigadores ${ }^{1}$ ) y los informantes ${ }^{2}$ que colaboran con los proyectos de investigación.

En este escrito se plantean dos cuestiones principales. Los componentes a considerar en la planificación y la conducción de las entrevistas, y los problemas de interpretación que pueden aparecer en este contexto. La perspectiva elegida proporciona herramientas que nos permiten (a los especialistas en música u otro lenguaje artístico) reconocer la complejidad que se esconde en los pliegues de la conversación y en la producción de un saber sobre la cultura de los grupos humanos estudiados o sobre la realización artística de una persona o un colectivo, entre otras temáticas recurrentes en los trabajos sobre la música.

La planificación e implementación de las entrevistas se relacionan necesariamente con el diseño de los proyectos de investigación, y con el posicionamiento del entrevistador con respecto a las relaciones de poder social que se establecen o configuran en la interacción con los informan-

1 Para favorecer la fluidez en la lectura utilizaré las formas masculinas de los sustantivos con valor inclusivo: investigadores, entrevistadores o entrevistados. Duplicar cada uno de estos términos para visibilizar la presencia femenina en dichos roles obstaculiza la comprensión de los conceptos y discusiones tratados en este texto. Asimismo, el uso de "les" o "XS" aún no es corriente en los escritos académicos. No obstante, manifiesto mi adhesión a la necesidad de incluir la perspectiva de género en esta clase de comunicaciones.

2 En la bibliografía sobre entrevistas se utiliza diferente terminología para referirse a las personas entrevistadas: informantes, entrevistados, nativos y colaboradores son nombres que aparecen en distintos textos de etnografía. 
tes. Considero que este es uno de los problemas más relevantes y menos discutidos en las investigaciones sobre música, musicología y etnomusicología. Me refiero a las investigaciones sobre las culturas musicales y sus articulaciones con las identidades de clase, de nacionalidad, de género, etcétera. En esta clase de investigaciones, los comentarios sobre las prácticas musicales develan las disputas simbólicas existentes en una comunidad o las que se concretan en acciones políticas generalmente autogestionadas. La habilidad del entrevistador consistirá en reconocer las tensiones políticas, ideológicas y sociales cuando no son expresadas abiertamente, cuando se hacen explícitas mediante expresiones no verbales. También es fundamental que esté informado sobre las luchas históricas que la comunidad estudiada sostiene y considera importantes, ya que en la entrevista se evocan las cuestiones del pasado y se actualizan sus significaciones.

Las investigaciones a las que me refiero recorren un amplio arco de intereses que abarcan desde, por ejemplo, la averiguación de quiénes toman las decisiones en un grupo de cumbia o en una producción discográfica, hasta trabajos que analizan la performance ${ }^{3}$ de Lady Gaga cuando cantó el himno de los Estados Unidos de Norteamérica durante la toma de posesión del presidente Joe Biden. En resumen, propongo un marco que contribuye a fundamentar los estudios sobre música desde una perspectiva crítica.

Las reflexiones propuestas forman parte de una investigación de mayor envergadura. Comencé a profundizar en las entrevistas a partir del trabajo con las fuentes documentales de los Archivos de Carlos Vega (Sánchez, 20I4). En mi tesis doctoral ${ }^{4}$, fundamenté la importan-

3 En mis investigaciones utilizo la definición de performance propuesta por un grupo de especialistas, que a partir de la década de 1970 redefinieron los estudios del Folklore como disciplina científica. La definición más difundida es la que elaboró Richard Bauman en base a los aportes de los estudiosos que compartían su trabajo sobre arte verbal (narrativa oral). Distingue la performance de otros eventos comunicativos por el modo artificioso que una expresión verbal adopta cuando tiene una intencionalidad estética. La performance como ejecución y actuación es definida como un "principio organizador" que comprende: la forma estética e incluye la acción de los actores (performers) y la realización de la obra artística, la situación social y el contexto de las ejecuciones, y el rol de los actores y la audiencia. Estos componentes son interdependientes, aunque para hacer posible el análisis se los examina o describe por separado.

4 Se puede consultar (en forma libre y gratuita) la tesis doctoral completa en versión digital; el enlace al repositorio de la UCA se indica en la bibliografía, en el apartado final de este trabajo. 
cia de la articulación del análisis de la música con el análisis de las entrevistas efectuadas en Jujuy por este etnomusicólogo y folklorista pionero (Sánchez, 20I8a). Los investigadores que en su producción intelectual ponen en diálogo el análisis morfológico de las obras, o el estilo interpretativo de un músico con algún aspecto del orden de lo societario parten de la premisa de que la música no «refleja» comportamientos o estructuras sociales sino que constituye la cultura; ya sea que se indague sobre el proceso compositivo o sobre la manera en que se negocian los intereses de los artistas (autores, intérpretes, arregladores, etc.) con los distintos mediadores del negocio de la música, en cualquiera de estos casos es interesante explorar y comparar lo que los distintos actores sociales opinan. La entrevista es una estrategia ideal para llevar a cabo proyectos en los que el investigador está dispuesto a sorprenderse de lo que encuentre en el "campo", aunque las circunstancias lo obliguen a introducir modificaciones en su proyecto.

Los músicos, musicólogos y folkloristas hacemos entrevistas y observaciones in situ. Esto significa que deberíamos reflexionar sobre la práctica profesional así como lo hacemos en el rol docente. La indagación en torno a las relaciones de poder y los roles asumidos o instaurados en el contexto de la entrevista se justifica porque, por una parte, nos interpela sobre el compromiso ético-político que asumimos. Las decisiones de los investigadores o entrevistadores inevitablemente orientan la producción de un saber hacia un lado u otro. Por otra parte, las convocatorias de revistas académicas como las de las asociaciones especializadas en estudios de música popular ${ }^{5}$ demuestran un marcado interés en los trabajos que examinan expresiones semióticamente complejas como las performances de música popular en celebraciones masivas como fueron las conmemoraciones de los Bicentenarios en América Latina (Sánchez, 2013). Teniendo en cuenta esta tendencia a establecer relaciones entre música, política y nuevas formas de participación ciudadana, hago una

5 Me refiero principalmente a las convocatorias de la Asociación Internacional para el Estudio de la Música Popular (IASPM-International Association for the Study of Popular Music) y a la Rama Latinoamericana de la misma institución (IASPM-AL). Con respecto a las publicaciones especializadas en música popular y etnomusicología, la Revista TRANS (arbitrada por la SIBE) es una de las más prestigiosas. Las convocatorias de estas instituciones están disponibles en línea y así se puede comprobar qué temáticas sobresalieron en las últimas décadas. 
aclaración: parto de la premisa de que los lectores de este trabajo son especialistas en artes, educación y humanidades; la formación principal de este colectivo no se centra en las técnicas de recolección de datos en ciencias sociales ni en los problemas que surgen en la interpretación de los datos obtenidos en el contexto de la entrevista ni profundizan en las relaciones de poder que se instauran entre el investigador y los entrevistados (todas estas temáticas son materia de reflexión en este escrito). Entiendo que la formación de los profesionales a quienes dirijo estas líneas se centra en los saberes propios del campo artístico y la docencia, en el desarrollo de las competencias de la especificidad (interpretación, composición, dirección, teoría musical, etc.) y en áreas troncales del proceso de enseñanza y aprendizaje como la didáctica y la pedagogía, entre otras. Si bien en asignaturas como Metodología de la Investigación se comparan definiciones de técnicas de recolección de datos como la entrevista y la encuesta, generalmente en estos espacios curriculares no se desarrollan discusiones fundamentadas sobre los enfoques más convenientes en entrevistas implementadas por músicos que no han accedido a una bibliografía exhaustiva acerca del análisis de las performances desde la perspectiva de la sociología de la música y la sociolingüística. Mi propia experiencia como investigadora en música popular y etnomusicología me hizo ver que es necesario insistir en la difusión de este enfoque. Los interesados en profundizar en la pertinencia de la perspectiva propuesta pueden consultar un trabajo anterior (Sánchez, 2018a: I29-I46).

Organizo este artículo en tres partes. En la primera, distingo los distintos tipos de entrevistas y especifico las características de las que se enmarcan en proyectos de investigación. En la segunda parte desarrollo las nociones claves; entre otras, las formas de registro de la información y la situación social en la que se lleva adelante el encuentro. Hago hincapié en las modificaciones y adaptaciones de los discursos sonoros y verbales en el proceso de entextualización, cuando las ejecuciones ${ }^{6}$ (per-

6 Performance es sinónimo de actuación o ejecución. Este concepto se refiere a comportamientos construidos culturalmente que tienen una historia social y generan reacciones específicas. Este entramado de significaciones y reacciones hace que, por ejemplo, un movimiento de pelvis de Sandro tenga una reacción en el público femenino y que a la vez, provoque la inmediata asociación con el estilo actoral de Elvis Presley. 
formances) verbales y musicales son convertidas en textos $^{7}$. En esa operación se seleccionan diálogos y lo vivenciado por los actores es relatado y representado desde el punto de vista del entrevistador o analista. Luego, presento ejemplos de entrevistas reales. Retomo los conceptos presentados y los aplico al análisis de distintas situaciones que se han presentado en casos concretos.

\section{$1 \cdot$ LAS ENTREVISTAS: PROPÓSITOS, FORMATOS Y ÁMBITOS PROFESIONALES}

La entrevista es un encuentro etnográfico y al mismo tiempo un evento discursivo. El producto resultante, el texto, es elaborado por investigadores y difundido por las instituciones académicas. Este punto de partida ha motivado la discusión acerca de las posiciones de poder social que se configuran en la entrevista. Poner la lupa en el proceso de producción de textualidad implica que se exploran las estrategias implementadas en la interacción con los entrevistados ${ }^{8}$ desde el momento inicial, previo a la entrevista en sí misma. Casi siempre hay agentes que intervienen en la vinculación entre el trabajador de campo (investigador o científico) y la persona entrevistada o el grupo estudiado. Los comportamientos de la comunidad (por ejemplo, la manera en que ciertos actores que ocupan posiciones de poder perciben a los investigadores y a los informantes) constituyen un tema clave en el estudio de las interacciones sociales.

La clase de dispositivo que implementan los músicos, musicólogos, sociólogos y antropólogos sociales se llama entrevista etnográfica. Se diferencia de otras entrevistas (médicas, laborales y las que realizan las instituciones educativas) porque de acuerdo con cada profesión se estable-

7 En principio, lo que sucede en las entrevistas se convierte en un texto extractado, por ejemplo los informes que se ajustan al formato adecuado para comunicar el avance de la investigación entre los académicos.

8 En la bibliografía de referencia sobre entrevistas, así como en el presente trabajo, los términos entrevistadores, investigadores y analistas son utilizados como sinónimos. Normalmente, la misma persona que dirige las entrevistas analiza los datos recabados en el trabajo de campo. Sin embargo, si examinamos punto por punto las tareas, la expertise y las responsabilidades que les corresponden a cada uno (entrevistadores, investigadores y analistas) encontraremos diferencias. 
cen diferentes objetivos, se recaba información de diferente índole, los datos son analizados con distinta metodología y, sobre todo, el proceso de entrevistar es sustancialmente distinto en cada caso.

En las entrevistas que nos interesan partimos de la idea de que la interacción entre los actores ${ }^{9}$ (entrevistado/s e investigador) y las posiciones de poder asumidas por las partes en este contexto se convierten en un objeto de estudio. Lo que señalo a continuación se relaciona con el recorte de la investigación y, por ende, con la epistemología del área de estudio.

Las entrevistas definidas como estrategias metodológicas en investigaciones sobre prácticas musicales se llaman entrevistas etnográficas. Se inscriben en la intersección entre las ciencias sociales, las humanidades, la historia y la etnografía, sin importar la tradición musical o el rótulo clasificatorio que se les asigne (popular, erudita, folklórica, etc.). Estas entrevistas se caracterizan por la profundización del análisis en las situaciones comunicativas y en los procesos subjetivos que los actores experimentan. Lógicamente, para generar este vinculo de confianza y disponer las condiciones que permitan producir un saber conjuntamente con los entrevistados (o informantes), el investigador, siempre que sea posible, propondrá una serie de entrevistas en profundidad.

La producción de un saber conjunto sobre lo que determinado compositor o músico o grupo siente, piensa y cree que la música le permite "hacer» en su vida cotidiana o en la gestión de sus conflictos y en la expresión de sus afectos exige que las entrevistas se planteen desde la perspectiva adecuada: el enfoque que estudia la diferencia entre discurso y texto, y que se ha ocupado de ponerle nombre a los componentes del evento comunicativo particular que es la entrevista.

La diferencia más elemental entre la entrevista etnográfica y, por ejemplo, las entrevistas médicas o laborales, consiste en que estas últimas se plantean como un acto de «apertura y cierre». Generalmente se estructuran como una ficha, y consisten en una serie de preguntas cuyas respuestas se limitan a un número acotado de opciones, y también se diferencian

9 La entrevista no es una conversación casual o espontánea, es un evento comunicativo particular en el que los participantes actúan ("performan») roles asumidos de manera implícita o que derivan de acuerdos previos. 
de la entrevista etnográfica porque se reducen a uno o dos intercambios verbales en los que se busca información estrictamente preestablecida.

En metodología de la investigación circulan diversas definiciones de la entrevista en ciencias sociales, dependiendo de la antigüedad del libro consultado y de la perspectiva de análisis que prevalezca en cada caso. Comúnmente se define la entrevista como una técnica de recolección de datos; sin embargo, no es una técnica que se pueda aprender de una vez y para siempre. La entrevista, entendida como práctica etnográfica y etnomusicológica, requiere ser conceptualizada con mayor profundidad.

Desde la óptica de la sociolingüística, la entrevista es una estrategia comunicativa a la que los investigadores recurren para obtener gran cantidad de información (datos) en el menor tiempo posible. Al comienzo de este apartado señalé que la entrevista es un evento discursivo particular, que tiene sus propias reglas de interacción social, ya que requiere de acuerdos previos sobre la duración de los encuentros, la ocasión y el lugar en el que estos eventos se llevarán adelante.

Cuando alguien menciona la palabra entrevista, automáticamente se piensa en la entrevista periodística típica de los programas radiales o televisivos. Este formato tiene propósitos relacionados con el mundo de los media. La idea de éxito depende de la medición de audiencia (rating), de la repercusión o el impacto que el contenido de la entrevista cause en las redes sociales, de la cantidad de reproducciones que logre alcanzar y la cantidad de tiempo que se mantenga vigente como noticia. La finalidad es la novedad; por ejemplo, la promoción de una nueva obra teatral o película sustenta la presencia de la personalidad entrevistada. Los intercambios verbales tienden a lo anecdótico y los temas de conversación se abordan de manera superficial ${ }^{10}$. En estos casos se ponen en juego intereses comerciales que orientan o determinan los objetivos y las normas de interacción entre los participantes. La información comunicada es evaluada principalmente por el entrevistador en el transcurso del encuentro, aunque si se trata de un programa "en vivo" pue-

10 Hay excepciones, por supuesto; en algunos ciclos televisivos las entrevistas se orientan hacia la producción de un saber de tipo pedagógico; por ejemplo, en Canal Encuentro, Canal (á) o en las entrevistas de programas radiales dependientes de universidades. 
den intervenir el productor o la audiencia provocando un cambio en el rumbo de la conversación; además, el producto puede ser editado antes de su publicación.

Estos criterios de realización y evaluación también son aplicables a las entrevistas que comparten los usuarios de YouTube, Instagram u otras plataformas. A diferencia de estas, las entrevistas que se enmarcan en investigaciones de musicología o de etnografía educativa asumen un compromiso ético y deben ajustarse a las regulaciones profesionales. En el ámbito académico, los resultados o informes de avances de una investigación están sujetos a distintas etapas de validación. En una palabra, es necesario demostrar que el proceso se cumplió en conformidad con los lineamientos consensuados por otros investigadores e instituciones evaluadoras. Por un lado, la información que surge de los estudios académicos, como las tesis doctorales o los informes publicados en revistas científicas, repercute en el desarrollo teórico-metodológico de las disciplinas. Por otra parte, es factible y esperable que ese nuevo saber sea utilizado para la toma de decisiones del sector o sectores vinculados a dicho estudio; me refiero a la posibilidad de modificar políticas en el plano institucional y social.

\section{$1.1 \cdot$ Diferentes diseños de entrevistas}

La elección del tipo de entrevista estará acorde al diseño de la investigación que puede ser cualitativo, cuantitativo o mixto. Las entrevistas se clasifican por su estructura y diseño en tres categorías: estructurada, semiestructurada y no estructurada (o entrevista en profundidad).

\subsection{1 $\cdot$ Estructurada}

En la entrevista estructurada, el entrevistador elabora un guión sobre la base de preguntas que se responden por «sí» o por «no» u ofrecen una serie limitada de respuestas. Se confecciona una lista ordenada de preguntas y el entrevistador es quien dirige el intercambio verbal. Las preguntas generalmente se ordenan en forma secuenciada. Se comienza 
con los datos personales del entrevistado y luego se hace foco en distintos aspectos del tema a explorar. La entrevista estructurada o dirigida también es conocida como entrevista con cédula (ficha). En este tipo de entrevistas las preguntas de los investigadores direccionan las respuestas que se obtienen (Guber, 2012).

Viene al caso comentar que este diseño fue utilizado en los estudios científicos de folklore y en las recolecciones de música y danza populares de tradición oral que se llevaron a cabo en la primera mitad del siglo xx. Las entrevistas que implementó Carlos Vega en sus más de treinta trabajos de campo musicológico ${ }^{11}$ son un buen ejemplo de este modelo (Sánchez, 20I8b). En aquel tiempo se prefería la entrevista estructurada, porque se buscaban comparar y clasificar el mayor número posible de «especies» musicales y danzas ${ }^{12}$; en consecuencia, predominaba el enfoque cuantitativo. Se argumentaba que de este modo se lograba mayor objetividad, y la objetividad era uno de los ideales de la ciencia positivista, aunque el avance científico demostró que esta meta era más una pretensión que una realidad.

En la etapa inaugural de los estudios de folklore y etnomusicología, que abarca desde comienzos del siglo xx hasta la década de 1970, los científicos «iban al campo» con una serie de preguntas preestablecidas. Si lo que averiguaban sobre la historia de un género musical no coincidía con su propia opinión, no se buscaba reflexionar conjuntamente con el informante para integrar ambos puntos de vista; en lugar de eso, se consideraba que las "desviaciones» de los resultados esperados eran ocasionadas por el entrevistado; por ejemplo, en el caso de que el interlocutor se alejara de las temáticas previstas o que la información discrepara con las ideas que el investigador defendía como consecuencia de su

\footnotetext{
11 Estas entrevistas fueron registradas en sus Cuadernos de Viajes y pueden consultarse en la sección "Archivos de Música popular, folklórica e indígena del Fondo Documental Carlos Vega", el sitio web del Instituto de Investigación Musicológica Carlos Vega de la Facultad de Artes y Ciencias Musicales de la UCA, disponible en: http://www.iimcv.org/ archivo_popular_fondo_vega.php.

12 Los estudios locales de música popular y folklore realizados por Carlos Vega tomaron como base el paradigma de las ciencias biológicas y la arqueología; por ende, se buscaba sistematizar la cuantificación. Los sistemas de medición y la comparación eran procedimientos coherentes con el diseño cuantitativo. En la Argentina, los estudios comparativos de "especies" o géneros musicales predominaron en las investigaciones de etnomusicología y folklore hasta la década de 1960. Los estudios norteamericanos de arte verbal que reformularon el campo fueron incorporándose lentamente a partir de la década de 1980.
} 
estudio. Destaco que en la entrevista estructurada el diálogo se centra en una temática externa, no se refiere a la entrevista propiamente dicha ni a la interacción entre los participantes y las posiciones de poder asumidas por las partes o sus puntos de vista.

\subsection{2 $\cdot$ Semiestructurada}

En la entrevista semiestructurada, el investigador prepara un guión sobre los temas que proyecta tratar. Sin embargo, la conversación con el entrevistado se desarrolla de forma natural y las preguntas son abiertas. El entrevistado o informante puede expresar sus opiniones, matizar sus respuestas e incluso desviarse del guión cuando desea comentar algo que cree importante explorar. Esta clase de instrumento es apropiado en investigaciones cualitativas en las que se busca que el entrevistado se explaye sobre sus experiencias. El entrevistador procurará conectar rápidamente los temas emergentes con el tema central que se propuso investigar. En resumen, es importante mantener la atención para repreguntar o pedirle al entrevistado que explique por qué asocia determinado relato, emoción o imagen con el tema planteado inicialmente.

\subsection{3 - Entrevista abierta o entrevista en profundidad}

La entrevista no estructurada, conocida como entrevista abierta o entrevista en profundidad, consiste en una serie de encuentros cara a cara (vis à vis) entre el entrevistador y el/los entrevistado/s. En este caso, «el entrevistado tiene la palabra», se le propone que se presente de la manera que prefiera y que hable de los temas que le parezcan relevantes que los académicos conozcan sobre su historia de vida o su cultura. La bibliografía especializada en etnografía indica que el entrevistador deberá ser receptivo a las temáticas que el entrevistado le proponga explorar y no forzará la situación para recabar datos sobre tópicos predeterminados. En esta situación, ¿el informante asume el rol de autoridad del saber?; ¡está preparada la academia para aceptar que la producción de un saber se realiza en forma conjunta con los entrevistados?

La entrevista en profundidad se caracteriza porque busca producir una etnografía densa, repleta de detalles y de reflexiones compartidas 
por los actores. Generalmente se complementa con la observación participante, la presencia (activa) del investigador en las actividades cotidianas a las que la comunidad lo invite.

A pesar de que la entrevista fue pensada originalmente como el producto de encuentros presenciales, con la invención de diversos dispositivos tecnológicos los encuentros mediados virtuales son cada vez más frecuentes y se han naturalizado otras maneras de entender qué es estar "frente a frente»; el advenimiento de la pandemia de covid-I9 nos invita a revisar esta definición tradicional. La pregunta que surge es, ¿la interacción en forma presencial es equiparable a una entrevista que transcurre mediada por un dispositivo de pantalla?

El siguiente comentario sobre el clima de la entrevista pone la lupa en lo decisiva que puede resultar la primera impresión en un encuentro presencial y, sobre todo, en la experiencia de lo que se siente «a flor de piel».

Es evidente que el trabajo de análisis e interpretación de una entrevista a profundidad comienza mucho antes de la propia grabación de la cinta. Las condiciones para el establecimiento de la relación de investigación son esenciales para devolver, si se quiere objetivar la relación entrevistador/entrevistado y comprender el desarrollo de la entrevista. Puede decirse, sin exagerar, que los primeros momentos del encuentro son estratégicos: marcan un clima, una «atmósfera» en la cual se desarrollará a continuación la entrevista. (Beaud, 20I8:I9I).

En síntesis, si los proyectos de investigación incluyen entrevistas, además del análisis estructural o morfológico de una obra, un estilo compositivo o una performance musical es preciso analizar las significaciones, los sentidos que estas prácticas tienen para los creadores y la comunidad (audiencia o consumidores, productores artísticos, discográficas, empresarios, políticos a cargo de organismos de cultura, etc.).

En investigaciones cualitativas o mixtas, las entrevistas coincidirán con los lineamientos teóricos de estos diseños, y necesariamente pondrán de relieve la potencialidad comunicativa que la música tiene. Para conocer de qué manera los actores experimentan la música, la vivencian 
cotidianamente y la crean o recrean, los investigadores recurrimos a las áreas del saber que se ocupan de estudiar la conexión entre el habla (la expresión oral), las relaciones humanas y las visiones de cada cultura o grupo social en el entendimiento del mundo. La Antropología Social, la Etno-historia, la Etnografía, la Sociolingüística conjuntamente con el Folklore (narrativa oral) y la Crítica Literaria han realizado desarrollos teóricos y estudios empíricos que convergen en la perspectiva cualitativa. Estas disciplinas han contribuido a que las entrevistas se conviertan en una especialidad dentro de las Ciencias Sociales y las Ciencias de la Comunicación.

\section{$2 \cdot$ Marco teórico}

La entrevista es un evento discursivo y lo que se comunica en ese contexto tiene efectos concretos en el plano individual y social. Por lo tanto, los enfoques de investigaciones sobre música que incluyen entrevistas son interdisciplinares.

Las investigaciones que se plantean desde perspectivas críticas ponen de relieve la agencia ${ }^{13}$ (agency), que es la capacidad que los actores tienen para modificar la realidad social, por ejemplo en la recreación de repertorios simbólicos y la actualización de las significaciones de sus comportamientos. Estos estudios buscan poner en diálogo lo que los músicos, representantes, fans, media, musicólogos, etc. creen, piensan y sienten que la música es, o lo que la música les permite «hacer» individual y colectivamente. En este punto, el discurso verbal es equiparable al discurso musical. Para una mayor precisión sobre la noción de performance desde la perspectiva de los estudios de arte verbal, puede consultarse la fundamental contribución de Richard Bauman, «Verbal Art as Performance» (Bauman, 1975).

13 La agencia (agency) es la gestión, la capacidad de actuar que los grupos sociales manifiestan para hacer conocer diferentes modos de comprender el mundo y reivindicar sus identificaciones con luchas étnicas, culturales, de género, entre otras. 
La perspectiva crítica en los estudios sobre música va más allá de la descripción de elementos técnico-expresivos de determinado género musical o estilo de ejecución instrumental. Además de estos asuntos, se busca develar el entramado relacional y la historia de los géneros en relación con el grupo o sociedad estudiados.

Los postulados teórico-metodológicos de la etnografía del habla tienen múltiples antecedentes en los estudios de música popular y folklore. El punto de partida es el modelo de análisis de los componentes del evento comunicativo propuesto por Roman Jakobson (I960), que fue expandido por Dell Hymes entre los años 1964 y 1972. En base a estos modelos, Charles Briggs (1986) desarrolla las nociones que trataremos en relación con las condiciones en las que se llevan adelante las entrevistas y los errores más frecuentes en el análisis de los datos.

El proceso de entrevistar es complicado, porque todo evento comunicativo es multidimensional, y aunque la información se transmite en distintos niveles o por diferentes canales simultáneamente, a los fines del análisis es preciso separar los problemas en partes conceptualmente tratables.

En los estudios de performance desde el enfoque socio-lingüístico, quien dirige la entrevista no solo está atento al registro de la conversación, sino que también evalúa lo que acontece en la comunicación gestual, cinética, proxémica, etc. A esta complejidad se suma el desafío de documentar las ejecuciones sonoras o musicales que pueden surgir de manera espontánea o como resultado de acuerdos previos.

A continuación, presento algunos conceptos claves: los objetivos interaccionales de las entrevistas, las formas de registro de la información, las normas de interacción, la situación social y la contextualización.

\section{$2.1 \cdot$ Los objetivos interaccionales}

Los objetivos interaccionales del investigador o entrevistador están claramente definidos de antemano. En cambio, los entrevistados podrían no tener tanta claridad respecto de las motivaciones que los impulsan a 
participar y colaborar en una investigación, o mejor dicho, sus objetivos podrían variar con el transcurso de los encuentros en la medida que se van interiorizando de la actividad o el alcance del proyecto. Habitualmente los entrevistados son personas que no pertenecen al ámbito académico o científico, y si bien pueden tener una idea de la finalidad de la entrevista, es factible que en el transcurso de la misma manifiesten dudas o prefieran hablar sobre temas diferentes de los que fueron establecidos de antemano. Los especialistas remarcan que una de las tareas mas difíciles para el investigador consiste en establecer la referencia y mantenerla mientras dura una entrevista. En el transcurso del diálogo frecuentemente se pierde la referencia, es decir que los participantes tienen dudas sobre qué asunto están hablando en determinado momento. Asimismo, surgen dudas sobre el modo en que deberían comportarse en el contexto de la entrevista, ya que a medida que se va generando un vínculo de confianza se van desdibujando los roles, por ejemplo, se corre el riesgo de que se torne en una relación amistosa. Por lo tanto, establecer y mantener la referencia a través de varios encuentros es aún más dificultoso.

Es indispensable que la construcción de la referencia o del marco de referencia sea una tarea conjunta entre el entrevistador y sus interlocutores, ya que se trata de construir sentidos y negociaciones ${ }^{14}$. Rosana Guber, siguiendo la línea teórica de Charles Briggs, ofrece otra definición de la entrevista etnográfica.

La etno-metodología ha sido una de las más fructíferas al advertir que la entrevista no es un ámbito o continente de donde se «extraen datos», sino una relación comunicativa y productiva de información especifica. La lógica de la entrevista no proviene del medio académico sino del proceso de conocimiento de la vida cotidiana. Ese proceso recon-

14 Los autores mencionados emplean el término negociación sin hacer aclaraciones sobre la índole de los tratos o pactos. Al profundizar en el estudio de la etnografía entendí que la negociación comprende diversas cuestiones, no solo se refiere a la negociación de las temáticas a tratar en la entrevistas y a las lógicas que los participantes pretenden imponer en el proceso de producción de un saber, además de estos asuntos, la negociación se refiere, entre otras cosas, a la retribución material o monetaria que el informante exige o que el entrevistador propone a cambio de información. 
figura el contexto y las fronteras entre el «adentro» y el «afuera» del encuentro (Guber, 1994: 30).

La entrevista ya no puede ser definida como «el arte de preguntar»; más bien debería ser identificada como el arte de establecer una referencia y mantenerla. Briggs remarca que el marco cognitivo, el sistema de creencias y los objetivos del entrevistador y los informantes o colaboradores en algunos casos son notablemente distintos.

La entrevista, entonces, requiere planificación y evaluaciones parciales de los resultados. Estas instancias permitirán reformular los objetivos, si fuese necesario hacerlo como consecuencia de los intereses o necesidades que expresen los entrevistados. La planificación supone un cierto orden en la formulación de las preguntas en el transcurso de un encuentro o una serie de encuentros, aunque el orden de las preguntas o las preguntas en sí mismas podrían modificarse de acuerdo con lo que acontece, lo que se va presentando en la interacción social.

\section{$2.2 \cdot$ El registro}

Todo evento discursivo es un fenómeno multifacético, y las entrevistas no son la excepción (Briggs, 1986: 39). En la entrevista se abren los canales físicos (principalmente el visual y el acústico), aunque también se deben tener en cuenta los canales psicológicos y no verbales que están presentes en todas las formas de comunicación en el encuentro interpersonal. Definitivamente, una de las tareas más arduas consiste en pensar de qué manera se registrarán los relatos y las formas de comunicación no verbales (gestual, proxémica ${ }^{15}$, etcétera). Pensar en los códigos adecuados para plasmar lo que se comunica gestualmente, los silencios y las variaciones de «tono» que se suceden en la conversación es un trabajo

15 Proxémica es el estudio del uso y percepción del espacio social y personal; la noción fue creada por el antropólogo Edward Hall en 1963. La comunicación proxémica se refiere a toda la comunicación del lenguaje corporal entre dos personas que hablan. Con el transcurso del tiempo, la terminología también se utiliza para aludir a la interacción de personas que se comunican de un modo no verbal, por ejemplo al danzar. 
subjetivo. En el registro escrito cada entrevistador utiliza los signos que considera apropiados para indicar, por ejemplo, las frases en las que su interlocutor hizo énfasis o mostró fastidio.

La complejidad de registrar lo que acontece durante un proceso de comunicación radica en la cantidad y diversidad de la información que se transmite en forma simultánea, de manera consciente e inconsciente. Por lo tanto, la previsión de los medios apropiados para documentar las performances (ejecuciones) verbales y sonoras que pudieran surgir espontáneamente o como consecuencia de las preguntas se convierte en una cuestión crucial. Cuando se entrevista a músicos, es muy probable que se realicen performances musicales de manera espontánea. Por lo tanto, se tomarán los recaudos para documentar adecuadamente los eventos que pudieran surgir. Es preciso planificar tanto el uso de los medios (audiovisuales, escritos, fonográficos y fotográficos) como la forma en que se harán los registros.

Las filmaciones contribuyen a captar gran parte de las actuaciones (performances) de los participantes, aunque a veces un mínimo gesto o una mirada significativos son percibidos únicamente por el entrevistador en un nivel intuitivo. Si se utilizan varias cámaras se captan mayor cantidad de detalles, aunque deben evaluarse pros y contras de esa multiplicidad, ya que la incorporación de terceros (encargados de filmar) podría distraer al entrevistado o afectar el «clima» del encuentro. La conveniencia de utilizar varios medios de registro a la vez puede incomodar o inhibir a las personas ${ }^{16}$.

La buena definición del sonido grabado y la minimización de interferencias del entorno permiten que la transcripción de la música y los diálogos sea completa. La calidad de los audios es particularmente importante en investigaciones sobre géneros musicales o que estudien el proceso creativo de compositores o el estilo de interpretación de un instrumento musical.

16 Si la entrevista incluye la presencia de público o representantes de artistas, otra persona debería filmar lo que hacen o dicen y luego, ese registro debería ser incorporado al análisis. No obstante, las decisiones que toman los responsables de hacer la filmación también son materia de análisis. El resultado depende de los acuerdos que previamente realicen el entrevistador y el encargado de la filmación. La edición de las imágenes también constituye una cuestión de capital importancia. 
Si las entrevistas se desarrollan mediante Skype, Zoom u otra aplicación similar, se tendrá en cuenta de qué manera el entorno tecnológico afecta las performances musicales y la interacción personal. Es evidente que la presencia de público incide en el ánimo del artista y en la manera en que transcurre un recital, especialmente cuando se trata de conciertos de música popular.

En la etapa siguiente al encuentro, en la tarea conocida como «la reconstrucción de la entrevista» a posteriori, el entrevistador reelabora el texto e incluye detalles sobre aspectos significativos que recuerde y que no haya podido registrar, por ejemplo, las expresiones no verbales del entrevistado, entre otras, los gestos, las miradas y la proxemia. La reconstrucción de la entrevista lleva a profundizar el análisis de lo acontecido, por ejemplo el investigador recordará que sospechó o detecto «faltas a la verdad» o contradicciones en las que el informante podría haber incurrido. También es el momento oportuno para comparar las diferentes versiones que le pudieron haber relatado sobre un caso determinado. De esta manera los apuntes registrados en el contexto de la entrevista se van articulando con las interpretaciones, aunque no es conveniente mezclar los registros originales con los desarrollos que surgen en la etapa de análisis de los datos.

Todo lo expuesto indica que el registro es tan importante como la metodología de análisis de los datos, pues estos instrumentos respaldan el informe que el investigador dará a conocer. Es recomendable utilizar una especie de planilla para registrar rápidamente los aspectos centrales que interesa documentar y que no siempre se hacen explícitos en la conversación.

Las descripciones de la dinámica del evento comunicativo, de la entrevista en sí misma, son fundamentales. La documentación de la valoración que el entrevistado y la audiencia hacen de las ejecuciones musicales que se dan en este contexto es un asunto elemental, pero también interesa averiguar de qué manera el informante o colaborador valora la experiencia compartida con el investigador. En síntesis, la indagación sobre la forma en que los agentes sociales modifican la estructura o la sonoridad de los géneros y modelan la recepción es una cuestión central en el 
estudio sobre la música. De la misma manera, es importante averiguar cómo los informantes construyen los marcos interpretativos en la valoración de una expresión musical determinada, cómo cambian las funciones de ciertos géneros según el ámbito en el que se ejecutan y cuáles son las disputas que surgen en torno a la forma, la sonoridad, etcétera. Por ejemplo, la performance musical en el contexto de un homenaje al Gauchito Gil bien podría articularse con las historias personales de los performers y develar identificaciones religiosas e identitarias, o posiciones ideológicas sustanciales.

\section{3 - Las normas de interacción, los roles y las posiciones de poder}

La entrevista es un evento discursivo singular. Se tendrá en cuenta que en este contexto los participantes son actores (performers) y en todo momento se ponen en juego los roles asumidos o implícitos. Dado que los actores participan de un complejo proceso en el que se genera «un saber", entrevistador $\mathrm{y}$ entrevistado/s intentan legitimar posicionamientos y discursos, porque, en definitiva, en la entrevista los actores representan posiciones de poder social. En el acto de manifestar su forma de comprender el mundo, de manera más o menos consciente, más o menos explícita, hay negociaciones de sentido. Las investigaciones de etnomusicología y musicología usualmente se exponen en congresos o se publican en revistas especializadas. Los estudios que sustentan este enfoque hacen énfasis en los procesos de generación de textualidad ${ }^{17}$, en la producción de narrativas sobre la música y los grupos humanos estudiados. Por lo tanto, el trabajador que hace entrevistas estará preparado para resolver los conflictos que pudieran surgir si no se respetaran los acuerdos sobre las normas que regulan estos encuentros.

Una de las instancias que puede generar desacuerdos es cuando los entrevistados olvidan o simulan que ignoran los objetivos interaccionales planteados al comenzar la entrevista. La decisión de sumar partici-

17 Amplío este punto en el apartado 3, referido a la contextualización. 
pantes también podría generar discrepancias; por ejemplo, si se trata de un estudio sobre los tambores del candombe, es bastante probable que el entrevistado requiera la participación de otros músicos para ejemplificar en qué consisten los «toques» de cada tambor y cuál es su función. Es importante definir un marco de actuación para que no se produzcan dispersiones o conflictos. Puede suceder que en el transcurso de la entrevista un allegado o conviviente del entrevistado intervenga por voluntad propia y que esta irrupción modifique el clima de intimidad o, por el contrario, que el informante permita su participación. Estos emergentes pueden cambiar el rumbo de la investigación de manera decisiva, ya que la información aportada por un tercero podría contradecir lo que dicen o piensan los actores originariamente consultados. Estas situaciones imprevistas generalmente conllevan a la redefinición del proyecto de investigación.

El contacto previo con los informantes ayuda a generar un clima de confianza entre las partes en los encuentros iniciales. No obstante, es posible que lo establecido en los acuerdos previos se modifique en el transcurso de la entrevista. Ha sucedido, por ejemplo, que el entrevistado, como anfitrión, insista en que el entrevistador o los participantes consuman alcohol durante la entrevista ${ }^{18}$. En el apartado 4, sobre ejemplos de entrevistas, amplío cuestiones de discrepancia entre los actores (entrevistador e informantes) que se han dado en situaciones reales.

Como en todo plan, se fija un lapso de tiempo estimado, pero hay que ser flexible en el caso de que la entrevista se dilate o se acorte. La administración del tiempo no depende solamente del acuerdo previo entre los participantes; a veces el silencio del entrevistado o un gesto de emoción puede ser el indicador de que es mejor dejar cerrar la sesión en ese punto.

También es conveniente acordar los temas sobre los que el entrevistado prefiere o está mejor predispuesto a conversar. En el contacto previo al encuentro, si se implementan entrevistas estructuradas, el entrevistador o investigador comunica o «adelanta» las preguntas que formulará.

18 El alcohol no es el único consumo significativo que puede resultar problemático en el contexto de la entrevista. 
En entrevistas no estructuradas, se pueden acordar las temáticas entre los actores. En todos los casos es conveniente pedir anticipadamente que el entrevistado seleccione fotos, instrumentos musicales, partituras y todo objeto que considere significativo para complementar su discurso o refrescar su memoria.

El lenguaje utilizado por el investigador establece el nivel de formalidad del evento. Al respecto, se han de considerar las convenciones que cada grupo social utiliza; por ejemplo, el uso del «don» o "dońa» sigue teniendo vigencia en ciertas comunidades. En cuanto a las convenciones sociales de la conversación, se tendrá en cuenta que en algunas culturas no se acostumbra mirar a los ojos a personas ajenas al grupo familiar o cercano. Asimismo, la proxemia es un aspecto por considerar, dado que el uso y la percepción del espacio social y personal varía de un grupo social a otro. El tono de la voz, el respeto por las pausas y el significado de los silencios son elementos esenciales para generar un clima de confianza que haga posible una comunicación fluida.

A veces el lenguaje técnico utilizado por los investigadores puede obstaculizar el intercambio de ideas. Generalmente la terminología y los conceptos que manejan los entrevistados difieren de los que manejan los analistas, especialmente si se trata de músicos populares o de un grupo de fans o de compositores que no se han formado en un ámbito académico; es indispensable conocer el repertorio de ideas que ellos utilizan para evaluar y valorar sus producciones y las de otros.

La manera en la que se expresan las ideas es otro punto sensible en la etnografía. Por un lado, es común que haya discrepancia entre los conceptos y la terminología específica utilizados por ambas partes; por ejemplo, el músico aficionado puede llamar ritmo a lo que el académico define como métrica. Sugiero disponer un apartado a manera de glosario para explicar el significado de la terminología que los informantes emplean. De este modo se evitan discusiones en el momento en que el entrevistado conoce el informe final del investigador y aprueba el contenido proporcionado en su colaboración. Respecto de las aclaraciones sobre las diferencias de interpretación de un hecho puntual, es fundamental que consten en el informe: la interpretación del analista no debe 
reemplazar lo que sus interlocutores manifestaron. Igualmente, las formas idiolectales de uso de la lengua o los regionalismos han de reproducirse tal como fueron dichos. La manera en que las expresiones verbales son reproducidas en los textos académicos que los investigadores difunden es una cuestión delicada porque atañe a la ética profesional y al vínculo de confianza entre los actores. Los reemplazos del repertorio verbal utilizado por los informantes por palabras que los académicos consideran más adecuadas, constituyen un ejemplo privilegiado para el análisis de las relaciones de poder. El entrevistador que se posiciona como autoridad del saber puede verse tentado a hacer reemplazos de las expresiones vertidas por sus informantes por considerarlas inapropiadas para reproducirlas en el ámbito en el que trabaja, o lo que es peor, imponer sus interpretaciones de lo que ha sido discutido por considerar que la lógica de su interlocutor es inconsistente. En cualquiera de estos casos, quien realiza el informe de investigación o analiza la entrevista incurre en un error. Impone su punto de vista en lugar de dar cuenta de las discrepancias existentes, y a la vez, desconoce todos los argumentos que la sociolingüística y la etnografía han construido para legitimar los modos de expresión de la «otredad».

Si aceptamos que, «... el lenguaje construye la situación de interacción y define el marco que le da sentido» (Guber, 20I2: 42), queda definitivamente claro a qué nos referimos cuando hablamos del alcance del evento discursivo como ejecución o performance.

En la introducción del presente escrito dejé entrever que la entrevista es un proceso de construcción de un saber social en forma colaborativa. Las expresiones de los entrevistados, así como la música que ejecutan, constituyen su cultura y resultan eficaces en la comunidad de pertenencia. Por ende, los enunciados no deben ser censurados o reformados, a menos que el protagonista prefiera cambiar o suprimir alguna frase u opinión cuando hace una revisión del texto final y autoriza su difusión. Es fructífero indagar acerca de la valoración que el informante o el grupo de referencia hacen de los vocablos o dichos que a quienes trabajamos en instituciones educativas nos resultan agraviantes o grose- 
ros. En definitiva, los estudios científicos no tienen propósitos moralizantes. La literatura y el arte tampoco tienen esa misión.

El tango contiene términos en lunfardo, que a veces son subidos de tono. Igualmente, los textos de la cumbia villera son cuestionables en muchos sentidos. Sin embargo, cuando estos repertorios son objeto de estudio, los analistas reproducen las expresiones como fueron escritas o grabadas en los discos ${ }^{19}$.

Es evidente que en el contexto de la entrevista se dirimen relaciones de poder. El discurso de los entrevistados no siempre puede transcribirse enteramente, y al convertirse en un texto sufre mutilaciones y cambios. Sin embargo, la fidelidad para con las expresiones de los participantes es una cuestión de ética profesional; además, la forma en que se representan los datos comunicados develan las posiciones de los actores. Inclusive si surgieran vocablos o ideas controvertibles, su edición será materia de discusión en el momento de la publicación.

La etnografía, entendida en su triple acepción como campo, método y reflexividad, nos revela que el lenguaje, nuestros relatos y descripciones constituyen la realidad que refieren. La siguiente cita toma como base el trabajo de Briggs (1986).

La presencia del investigador constituye las situaciones de interacción, como el lenguaje constituye la realidad. El investigador se convierte, entonces, en el principal instrumento de investigación y producción de conocimientos (Guber, 20I2: 45).

Cuando Briggs y Guber remarcan que el investigador se convierte en el principal instrumento de investigación y producción de conocimientos, refuerzan la idea de que la interacción entre entrevistador y entrevistado se convierten en un objeto de estudio que no está escindido del

19 En jornadas académicas y congresos he observado que los expositores reproducen los vocablos o expresiones tal como son emitidos (en la entrevista) por los actores de las prácticas populares que estudian. En los estudios sobre la cumbia villera se analizan los textos de las entrevistas y las letras de canciones para explicar los conflictos de clase, etnia y género. A pesar de que la terminología resulta repudiable en el medio académico, los especialistas en ciencias sociales, comunicación y literatura argentina no reemplazan esas palabras que incomodan por otras, sino que profundizan en la significación y en la actualización de los sentidos que asumen las expresiones en su contexto. 
tema central que se propone en el título de un proyecto de investigación. En cierta forma, la reflexión del investigador sobre su propia actuación y el lugar que les asigna a sus informantes o colaboradores constituyen los temas elementales en la perspectiva crítica.

\section{$2.4 \cdot$ La situación social}

En el modelo de análisis de entrevistas de Charles Briggs, la definición de la situación social se refiere al momento en el que se lleva adelante el encuentro. El tiempo incluye la especificación del día de la semana, el momento del año o la estación; y la localización en la que la entrevista se desarrolla implica determinar si la entrevista se planteará como una actividad principal o si se desarrollará mientras transcurre otra actividad, por ejemplo, un espectáculo, un casamiento u otra ceremonia significativa para la familia o la comunidad. La descripción del entorno es fundamental, ya que el estado anímico del entrevistado varía ostensiblemente, así como también las respuestas serán diferentes dependiendo de que la conversación se desarrolle en un clima de intimidad o que oyentes indiscretos paseen por el lugar. El estado anímico o psicológico que el entrevistado atraviese también puede variar según su situación personal; por ejemplo, si la entrevista se realiza una vez finalizada su actuación escénica, la predisposición de un músico o bailarín será muy diferente de la que demostrará en momentos o partes del año en los que no ha estado trabajando.

\section{$3 \cdot$ Contextualización y Marco}

En el habla corriente, se utiliza «contexto» para referirse a situaciones diversas. Esta palabra es poco específica y a menudo dudamos sobre su alcance. En algunos trabajos académicos incluso se habla de contexto en relación con múltiples aspectos del objeto de estudio, como «contexto comunicacional», «contexto sociocultural», «contexto situacional», «con- 
texto institucional». Esta manera de interpretar el contexto, como si se tratara de estratos de algo estable que se puede describir objetivamente, ha sido superada. El contexto tampoco es el «telón de fondo» de determinada situación en la que se desarrolla la acción (Bauman y Briggs, 1990). Siguiendo la misma línea de argumentación, Kwabena Nketia ${ }^{20}$ comenta que sus estudiantes se referían al contexto representándolo como una sumatoria de factores («factor histórico», "factor cultural», «factor tecnológico», etcétera). En su crítica a las nociones de contexto y contextualización, explica que la representación factorial del contexto es inapropiada, porque procura describir aspectos de la realidad como si fueran aspectos del mundo material, «cosificables» (Nketia, 1990).

Desde la década de 1970, los estudios norteamericanos de folklore y arte verbal (verbal art) han señalado que el contexto en el que se produce una performance verbal o se registra una ejecución musical se configura como un entramado de significaciones y relaciones, que no puede ser pensado de manera separada del discurso producido en determinada situación social ${ }^{21}$. De igual manera, los textos generados por los investigadores o analistas a raíz de una entrevista o de la observación de una performance musical son una producción distinta del discurso verbal, gestual o sonoro al que se refieren. Cuando un discurso es descentrado de su contexto original y es centrado en otro, pasa por un proceso de entextualización. En la elaboración de un informe de investigación los discursos son transformados en textos adaptados a las expectativas y los objetivos de otros colegas (Bauman y Briggs, I990); por ejemplo, si sobre la base de una performance musical se elabora una partitura, ese evento comunicativo ha sido descontextualizado. El discurso es extraído de un contexto, es descentrado y recentrado en otro, en el contexto del estudio académico. En esa operación, sus funciones comunicativas y sus funciones sociales se modifican.

20 Nketia se formó en lingüística, antropología social y música. El artículo citado es el que resulta más esclarecedor para los investigadores, porque en él habla posicionándose desde la etnomusicología.

21 Por ejemplo, el estudio de cierto estilo de cumbia, el contenido literario, el baile o la sonoridad son importantes, pero el entramado de significaciones que informa sobre las disputas latentes reviste el mismo interés; por ejemplo, se incluirán preguntas que apunten a la forma en que negocian sus diferencias los subgrupos asociados a una clase social o que se autoperciben como un sector étnico particular; por ejemplo, los afroporteños y sus prácticas musicales. 
Destaco que el marco es la adaptación metacomunicativa del texto recontextualizado. El marco se relaciona con la forma en que el discurso verbal o sonoro (ejecutado "en vivo») fue convertido en un texto que resulte útil para los destinatarios; por ejemplo si una conversación en el contexto de la entrevista se reduce a un informe para compartir entre investigadores, la selección de párrafos que el estudioso haría probablemente no coincidirá con la selección que los músicos entrevistados elegirían si se les permitiera hacerlo.

La adaptación de una ejecución musical a una partitura implica la creación de nuevos textos; en ese pasaje, indefectiblemente se pierden y agregan datos. Procedimientos tales como la modificación de la duración o la altura de algunos sonidos que suelen hacerse para que la música grabada en una entrevista se ajuste al código de lectoescritura convencional que los académicos usamos en la transcripción, deben constar en el análisis. El marco es el conjunto de estrategias que se aplican para representar una ejecución en un texto.

Los llamados «estudios de contexto» cuentan con una arraigada historia en los estudios de etnografía del habla, sociolingüística y en los estudios de folklore norteamericano que incorporan la etnomusicología. En la década de 1970 se dejó de hablar de contexto para hablar de contextualización (Bauman, 1975; Bauman y Briggs, 1990). Al mismo tiempo, los estudios de ejecución o performance se consolidaron en el medio de un proceso de reformulación radical del debate sobre "texto" y «contexto». Antes de la década de 1980 era bastante común hablar de "contexto de uso» de determinadas prácticas musicales o de géneros de narrativa oral. En la actualidad, pensar texto y contexto como si fueran dos caras de una moneda o como una relación entre dos términos equivalentes no es apropiado. Desde entonces, se ha superado la idea reificada de contexto; la discusión se centró en la contextualización como proceso y en la generación de textualidad (Bauman y Briggs, 1996).

La contextualización implica un proceso activo de negociación en el cual los participantes examinan reflexivamente el discurso a medida que este surge, incrustando juicios sobre su estructura y significado en el habla 
misma. Los ejecutantes (performers) amplían los juicios al incluir predicciones sobre cómo la competencia comunicativa, las historias personales y las identidades sociales de los interlocutores modelan la percepción de lo dicho (Bauman y Briggs, 1990: 69).

Esta cita deja en claro que el estudio de performances se centra en la contextualización, y que se corrió el eje de análisis hacia los detalles contextuales. El «contexto» se refiere a la dinámica de las situaciones comunicacionales en las que se producen negociaciones de sentidos. El énfasis se trasladó del producto, del contenido de las performances verbales, al proceso, y de la descripción de las estructuras convencionalizadas a la agencia de los actores o cultores de determinados géneros (géneros musicales, de narrativa oral, rituales, etcétera). Entonces, lo que hacemos los investigadores es analizar performances musicales contextualizadas. Lo esperable es hacerlo conjuntamente con nuestros interlocutores para develar las leyes que regulan esas actuaciones y las maneras en que los actores actualizan sus significaciones de acuerdo con el tipo de situación social en la que la performance es representada. En definitiva, el contexto no es la descripción de un marco de referencia de lo que ocurre «ahí afuera».

En la definición de la configuración del contexto de las entrevistas, el investigador deberá reconocer las "pistas» de contextualización. Los géneros, en todos los casos, contienen formas de ejecución y modos culturalmente marcados; por ejemplo, en un concierto de música clásica, la audiencia tiene una expectativa respecto del repertorio, el sonido, la manera en que el público participa, el lugar físico en el que se lleva a cabo una actuación de este tipo, entre otros factores. Cuando se trata de performances de música ritual o de ceremonias que incluyen ejecuciones musicales, o de un recital de rock, además de estos se analizan otros aspectos que convergen para que la comunicación sea eficaz. En estos casos, el investigador o analista debe conocer en profundidad los códigos de interacción, y no basta con que los participantes hablen el mismo idioma. Las actuaciones artístico-musicales son multidimensionales. Para estudiarlas no es suficiente con describirlas, es preciso ahon- 
dar en su complejidad semiótica y en la dimensión comunicativa. En lo concerniente a la música popular ${ }^{22}$, el estudio implica tanto el rol de la industria del entretenimiento como el rol de los medios y los fans, entre muchos otros ángulos de análisis (Frith, 2014).

El investigador está obligado a desarrollar la competencia comunicativa en los términos consensuados en una comunidad; dichos términos establecen, por ejemplo, en qué momento se puede hablar, en qué tono se puede preguntar, a quiénes se puede interrogar dependiendo de su posición social en el grupo de pertenencia o de su edad. Briggs explica que cuando hizo entrevistas en México, a pesar de que dominaba el idioma local, debió aprender sobre qué tópicos se permitía a los forasteros preguntar, dado que se veía como una insolencia que interrogara sobre otro conjunto de temas (Briggs, 1986). Describe un ejemplo en el que el entrevistado debe asumir el rol de «alguien que no sabe» para que no fracase la investigación, incluso cuando había permanecido en el grupo durante varios meses. Con este comentario, por una parte, se remarca que la conversación es un acto político y cultural en el que se actúan las posiciones de poder social en tensión. Por otra parte, Briggs enfatiza que el entrevistador siempre ha de averiguar cuáles son las normas de conversación consensuadas en la comunidad que estudia; asimismo, investigará los criterios que sus interlocutores emplean en la evaluación de las competencias comunicativas. Ciertamente, las categorías que los académicos empleamos para valorar las expresiones musicales y los comportamientos interaccionales no son equivalentes a las que establecen los cultores de ciertas tradiciones o determinadas creencias religiosas, o en comunidades donde se reverencia la sabiduría o la autoridad que los ancianos tienen.

Dado que la entrevista es un evento discursivo, es indispensable tener clara la diferencia entre discurso y texto. El discurso, a diferencia del texto, es una unidad lingüística, sonora, musical o performativa: es una experiencia totalizadora que se desarrolla en un contexto.

22 Hablo de la música popular moderna, urbana, mediatizada, mediada, producida con intención comercial. Antecedentes sobre el enfoque de esta área de estudios y los debates de interés se encuentran en las actas de los congresos de la IASPM, asociación creada en 1981. 
El texto (recontextualizado) que el investigador produce es susceptible de manipulación; por ejemplo, en una partitura se utiliza un código y un marco que proviene del contexto académico. Es deseable que investigador e informante/s participen en la evaluación de los textos, ya que esta instancia casi siempre revela información sustancial sobre el objeto de estudio.

En la redacción de la metodología de la investigación, así como también en la comunicación de los informes de avance de un proyecto, es de capital importancia establecer la diferencia entre discurso y texto. A veces, quizás por la necesidad de condensar la redacción de los informes o presentaciones en congresos, los investigadores omiten hacer esta diferenciación, y el público podría pensar que hay una analogía entre discurso y texto.

\section{$4 \cdot$ EJEMPLOS DE SITUACIONES DE ENTREVISTAS}

Las situaciones que comento a continuación dan cuenta de los imprevistos o problemas que pueden surgir en las entrevistas y que cambian en algún aspecto el plan de los investigadores.

Miguel Ángel García se refiere a las primeras recolecciones de música popular realizadas por Robert Lehmann-Nitsche en La Plata (Argentina), a principios del siglo pasado, en el I900. Destaca que cuando escuchó las grabaciones fonográficas le resultó significativo el «ambiente de carácter festivo, cargado de chanzas y bullicio ${ }^{23}$ en que las mismas se llevaron adelante. García comenta que, a pesar de que los encuentros se realizaban en habitaciones de la vivienda que este antropólogo alquilaba, los indicios lo conducen a presumir que el científico germano no estaba presente durante las sesiones de grabación o, al menos, se ausentaba con regularidad. El contenido de los registros sonoros y lo relatado por Lehmann-Nitsche en un manuscrito sustentan su sospecha. En este documento, expresa que los jóvenes que se ofrecieron para colaborar como

23 García hace estos comentarios a partir de la escucha de los cilindros (originales) grabados por Lehmann-Nitsche y la consulta de los manuscritos del archivo. 
cantores en su investigación eran conocidos por su buena voz y talento. El grupo de músicos, de ambos sexos y de entre 17 y 30 años, concurría a su vivienda en el horario de la tarde y noche, porque durante el día los músicos trabajaban. Poco a poco, ellos empezaron a llevar amigos y comenzaron las discusiones políticas, aunque estas se hallaban «estrictamente prohibidas» por el recolector. Dado que la situación se volvió inmanejable para él, Lehmann-Nitsche resolvió dar por terminada la colección de fonogramas (García, 2006: 42).

El relato textual muestra la imposibilidad del investigador de mantener el acuerdo de las pautas de comportamiento establecidas con los músicos-informantes, quienes transformaron esos encuentros en reuniones de amigos. También es probable que estos jóvenes no tuvieran claridad sobre los objetivos interaccionales de las entrevistas, ya que las grabaciones debían estar exentas de interferencias para que fuera posible la transcripción y el análisis de las melodías ${ }^{24}$. Por un lado, se puede interpretar que los informantes no comprendieron el tipo de evento comunicativo en el que estaban participando. Por otro, surge el interrogante sobre las razones por las cuales Lehmann-Nitsche no cambió el lugar del encuentro o no limitó la asistencia de participantes, sino que prefirió interrumpir la recolección.

Otros colegas etnomusicólogos me han relatado situaciones similares. Se han visto obligados a interrumpir la entrevista, ya sea porque los informantes empiezan a beber alcohol o los reciben en estado de ebriedad, o les exigen (a los investigadores) que los acompañen con la bebida durante la conversación. Si bien esta clase de problemas perturba el desarrollo de la conversación e influye en los roles, el investigador debe tener en cuenta que comportamientos tales como el consumo de bebidas alcohólicas constituyen, en algunos grupos, una demostración de afinidad (rapport); por ejemplo, en comunidades que viven en zonas de clima muy frío, o en entrevistas que se desarrollan en tiempos de carnaval.

Otro ejemplo. Una etnomusicóloga interesada en estudiar el cuarteto cordobés consiguió una entrevista con el cantante Juan Carlos Jiménez

24 Estos procedimientos eran fundamentales en las recolecciones musicales, porque la finalidad era la comparación y clasificación de los ejemplos, y el bullicio invalidaba las muestras obtenidas. 
Rufino, más conocido como «La Mona». Para sacar adelante su trabajo, la colega no solo tuvo que mostrar que podía integrarse ejecutando un tema en su instrumento, sino que también debió compartir el vértigo de las giras de los fines de semana con el grupo, yendo de un local de baile a otro. A pesar de los riesgos que estos emergentes implican, indudablemente conocer la trastienda de la actividad aporta una experiencia de valor para el trabajo académico.

El tercer ejemplo se refiere al marco cognitivo y al modo en que se expresan las ideas. Se tendrá en cuenta que la mayoría de los músicos populares tienen un dominio empírico de la teoría musical. Si esto sucede, es muy probable que el entrevistado prefiera responder una pregunta o explicar un concepto mediante la ejecución de un instrumento o cantando. Por ejemplo, en un grupo dedicado al candombe, ante la pregunta: «¿Cómo aprendiste a tocar este tambor?», la respuesta de mi interlocutora fue: «Tocando». El director del grupo, a quien llamaban Artigas, «me colgó» el tambor y con un gesto me indicó que lo imitara. A partir de entonces, asistí a los ensayos. Debía dejar de preguntar y tomar nota. La manera en que ese grupo de candombe se comunica es tocando o bailando en el momento preciso. Aunque se trataba de un espacio público donde se enseñaba música, existía un acuerdo implícito en no admitir intrusos y no interrumpir la actividad por ningún motivo. Las preguntas eran respondidas con demostraciones empíricas, con gestos u obligando a que se marche mientras se tocaba el tambor. Al contrario de lo que acostumbramos a hacer los investigadores, se evitaban las descripciones o comentarios.

Después de varios meses de compartir regularmente las actividades con el grupo, estuve en condiciones de obtener información, aunque por inferencia y experiencia ya conocía parte de las reglas de interacción, las jerarquías y las relaciones de poder instauradas. En síntesis, los roles y la dinámica planificada cambió. Si bien mis informantes no expresaron la negativa a ser entrevistados, en la práctica me convocaron al espacio social en el que ellos eran anfitriones y la actividad consistía en la ejecución musical desde el principio hasta el final; los intercambios verbales eran prácticamente nulos. Con esto quiero decir que se debe estar dis- 
puesto a conocer los códigos sonoros y verbales y las pautas estipuladas por la comunidad estudiada.

Dado que mi investigación tenía como objetivo la realización de un trabajo para aprobar una asignatura en la carrera de instrumentista en música popular, lo que me resultó más complicado fue encontrar la información adecuada para adaptar la ejecución al formato requerido, para darle un marco (la adaptación metacomunicativa) al texto que debía presentar en la institución. Para ello, debí hacer una investigación aparte. Debí «traducir» los códigos musicales y gestuales de la performance al formato de la monografía, que contiene información escrita y gráfica.

\section{$5 \cdot$ ReFLeXión FinAL}

En relación con la inclusión de informantes, a pedido del entrevistado, en calidad de "colaboradores», se tendrá en cuenta que esta variante puede cambiar el rumbo de la investigación de manera decisiva. La información aportada por un tercero podría contradecir lo que dicen o piensan los entrevistados originalmente elegidos. En este caso sugiero incluir un apartado dedicado a la interpretación de las contradicciones o discusiones que surjan. De todos modos, las argumentaciones contrarias son de un enorme valor para mostrar las diferentes interpretaciones que los informantes hacen sobre un mismo hecho, y también conducen a visibilizar las diversas creencias y posiciones ideológicas de los integrantes de la comunidad estudiada.

Usualmente, en el proyecto el investigador fundamenta las razones por las que elige a los entrevistados. La tradición indica que aquel debe especificar si son informantes «calificados» o «no calificados». Sin embargo, esta manera de clasificar y calificar a las personas que colaboran con las investigaciones es, a mi entender, bastante cuestionable, porque he comprobado que esta práctica se repite "por inercia» desde principios del siglo pasado, y además, los etnomusicólogos de ese tiempo no explicaban los criterios aplicados en esta selección que se efectuaba de forma unilateral. 
Cabe aclarar que, por ejemplo, el vínculo de parentesco, amistad o convivencia no necesariamente justifica la inclusión de una persona en la entrevista. Esta clase de vínculo no convierte a los parientes y amigos en informantes calificados. A veces, las anécdotas pueden aportar algún dato desconocido, pero lo que interesa es la solidez de la argumentación cuando se hacen valoraciones acerca de una práctica, una obra o un artista. También es importante considerar que un informante calificado puede no tener formación musical institucionalizada, pero su experiencia como oyente y/o asistente asiduo a recitales justifica que se lo trate como un especialista en el tema. El informante calificado, por ejemplo, puede ser un coleccionista, y su aporte como oyente familiarizado con un género o la información que tiene sobre la historia de una orquesta de tango puede ser relevante. Igualmente, los integrantes de clubes de fans, por el hecho de seguir a un artista o una banda desde los comienzos de su carrera, tienen cierta autoridad como críticos y, además, conocen como nadie las disputas que se dirimen por las redes sociales. Para una profundización del problema del valor en la música popular, el sentido común y el lenguaje de la crítica, entre otros temas, recomiendo consultar los trabajos de Simon Frith (Frith, 20I4).

Las reflexiones vertidas se refieren a entrevistas dirigidas a actores que comparten el mismo código lingüístico-cultural y una posición o clase social similar. No obstante, este supuesto previo suele ser engañoso. En investigaciones que incluyan entrevistas a personas o comunidades en las que el margen de alteridad entre los actores es marcado, además del enfoque señalado se ha de considerar la perspectiva teórica de la etnografía con base en la antropología social.

En su libro sobre cómo aprender a hacer preguntas e interpretar los datos recabados, Briggs dedica un capítulo entero a los diferentes problemas comunicativos que atentan contra el éxito de las entrevistas. Remarca que el desconocimiento de los repertorios nativos metacomunicativos incide en la interpretación de los datos por parte del entrevistador y, en un elevado porcentaje de casos, estos «errores» invalidarían los resultados de la investigación. Los errores de análisis surgen cuando el investigador desconoce la significación que el grupo estudiado le otorga 
a determinadas palabras, tonos de voz y gestos. Aunque el entrevistador domine relativamente bien el idioma de sus interlocutores, si no conoce en profundidad las normas de conversación y las competencias comunicativas que rigen en la comunidad, es probable que se malogre el vínculo y que los informantes decidan no continuar con los encuentros (Briggs, 1986: 39-60).

En la misma línea de argumentación, el enfoque deconstructivista de la etnografía remarca que el stock de conocimientos del letrado o el científico es inseparable de sus representaciones del mundo, de sus creencias y de los textos que produce. Las lógicas con las que el investigador explica la realidad, así como sus preferencias estéticas y sus orientaciones morales e ideológicas, no pueden soslayarse. Lo ideal es que el investigador/analista haga explícito su posicionamiento subjetivo, el marco cognitivo desde donde formula los análisis de las culturas musicales y de los grupos humanos.

Uno de los problemas más usuales es la dificultad para establecer la referencia y mantenerla durante el proceso de la entrevista. En especial cuando los entrevistados se autoperciben con poder o ejercen un rol dominante en su grupo, o perciben al entrevistador como alguien que no conoce su cultura o desconoce el tema sobre el que investiga. Además, Briggs menciona que en comunidades mexicanas tradicionalistas están vigentes ciertas pautas para dirigirse a las personas de edad avanzada, a los ancianos. Las normas de conversación de algunas comunidades indican que no es usual mantener el contacto visual con los forasteros.

Finalmente, la entrevista no reemplaza la experiencia que el investigador, el analista o el entrevistador incorpora mediante un conjunto de vivencias compartidas con el grupo de seres humanos que participa de las prácticas que constituyen su tema de estudio. 


\section{REFERENCIAS BIBLIOGRÁFICAS}

BAUMAN, RICHARD (1975): "Verbal Art as Performance" en American

Anthropologist $\mathrm{N}^{\circ} 77$, Austin, University of Texas, pp. 290-311.

BAUMAN, RICHARD Y CHARLES BRIGGS (1990): "Poetics and performance as critical perspectives on Language and Social Life" en Annual Review of Anthropology $\mathrm{N}^{\circ} 19$, pp. 59-88.

BAUMAN, RICHARD Y CHARLES BRIGGS (1996): "Género, intertextualidad y poder social» en Revista de Investigaciones Folklóricas, Vol. 11, Buenos Aires, pp. 78-108.

BEAUD, STÉPHANE (2018): “El uso de la entrevista en las ciencias sociales. En defensa de la "entrevista etnográfica" en Revista Colombiana de Antropología Vol. 54, No 1, pp.175-218.

http://www.scielo.org.co/pdf/rcan/v54n1/0486-6525-rcan-54-01-00175.pdf [Consultado: 20/1/2021]

BEN AMOS, DAN (1995): “Hacia una definición de folklore en contexto" en Narrativa Folklórica (II). Introducción y compilación de textos, Buenos Aires, Fundación Argentina de Antropología, pp. 38-60.

BRIGGS, CHARLES (1986): Learning how to Ask. A Sociolinguistic Appraisal of the role of the Interview in Social Science research, Cambridge, Cambridge University Press.

FRITH, SIMON (2014): Ritos de la interpretación. Sobre el valor de la música popular, Buenos Aires, Paidós.

GARCÍA, MIGUEL A. (2006): "Una narrativa canónica de la música popular: a 100 años de las grabaciones de Robert Lehmann-Nietsche» en Revista Argentina de Musicología № 7, Buenos Aires, Asociación Argentina de Musicología, pp. 35-51.

http://ojs.aamusicologia.org.ar/index.php/ram/article/view/171/166 [Consultado: 23/2/2021]

GUBER, RosANA (2012): La etnografía. Método, campo y reflexividad, Buenos Aires, Siglo Veintiuno.

NKETIA, J. H. KWABENA (1990): "Contextual Strategies of Inquiry and Sistematization" en Journal of the Ethnomusicology, vol. 34, № 1 , Bloomington, Indiana University, pp. 75-97.

SÁNCHEZ, NANCY M. (2013): "La performance de Soledad Pastorutti en el Bicentenario Argentino: una versión coya power del carnavalito" en Vargas, Herom, et al. (eds.), Enfoques interdisciplinarios sobre músicas populares en Latinoamérica: retrospectivas, perspectivas, críticas y propuestas. Actas del $x$ Congreso de la IASPM-AL. Montevideo: IASPM-AL/CIAMEN, pp.168-181. http://iaspmal.com/index.php/2016/03/02/actas-x-congreso/ [Consultado: 12/3/2021] 
(2014): "Los cuadernos y las fichas de viaje de Carlos Vega (1931-1951). Digitalización de las Fichas del Fondo Documental Carlos Vega del IIMCV UCA y de los Cuadernos del Archivo Científico del INM para la creación de una red hipenvincular» en Revista del Instituto de Investigación Musicológica Carlos Vega № 28, Buenos Aires, Facultad de Artes y Ciencias Musicales de la Universidad Católica Argentina, pp. 363-374.https://repositorio.uca.edu.ar/ handle/123456789/1030 [Consultado: 14/3/2021]

(2018a): El carnaval "antiguo" y el carnavalito "moderno" documentados por Carlos Vega en la Puna y la Quebrada de Humahuaca (Jujuy): Constantes rítmicas, métricas y fraseológicas de un repertorio tradicional grabado in situ (1931-1945), EDUCA [serie Tesis doctorales], Buenos Aires. https://repositorio. uca.edu.ar/handle/123456789/9222 [Consultado: 13/3/2021]

(2018b): «Una etnografía de archivo sobre los trabajos de campo de Carlos Vega". Cuadernos de Folklore II, Buenos Aires, Academia Nacional del Folklore pp. 41-47. 